\title{
Quality of Life with Mental Health HIV-Infected Patients in India before and After Antiretroviral Therapy (ART)
}

\author{
Vinay Malik*, A.P. Garg, Tung Virsingh Arya and Maya Datt Joshi \\ LLRM Medical College and Hospital, Meerut, (UP), India \\ Shobhit Deemed University, Meerut, (UP), India \\ *Corresponding author
}

\begin{abstract}
AIDS/HIV is a chronic pandemic disease with significant morbidity and mortality. Although with the advent of HAART an increase in life expectancy and improved QoL has been noticed, evidences regarding prevalence of mental disorders among PLHIV are largely ambiguous. The present study aims to systematically review the evidences about the mental complications in PLHIV before and after HAART therapy. Literature search was conducted for past ten years (i.e. from 2008 to 2019) using the electronic data bases like PubMed, Googlescholar, Google using combination of keywords "AIDS" OR "HIV" AND "quality of life" OR "health related quality of life" AND "neurocognition", OR "cognitive deficit" OR "psychological complications" OR "mental health" OR "cognitive impairment" OR "depression" OR "anxiety" OR "dementia" AND "randomized controlled trials" OR "randomized clinical trials", OR "cohort study", OR "meta-analysis", OR "systemic review" OR "study". A total of 27 studies were included in the current systematic review in which 25 were cross sectional studies and 2 were reviews. These 27 studies recorded the neuropsychological variables either as a component of HRQoL/QoL or through usage of specific tools for assessment of depression, anxiety and HAND. Overall based upon the included studies it is evident that the enhanced coexistence of depression, anxiety and HAND with AIDS/HIV is common worldwide. Further, it was observed that the role of HAART in reducing the prevalence of neuropsychological disorders with disease progression is largely meager. It is recommended that baseline assessment of HRQoL, immune markers, and neuropsychological disorders may serve as better treatment strategy with improved outcomes. Further, considering the serious repercussions of mental disorders on HRQoL, it would be beneficial to incorporate additional treatment regimen for them in addition to HAART from the beginning.
\end{abstract}

Keywords

Mental Health, HIV-Infected patients, Antiretroviral Therapy

Article Info

Accepted:

24 May 2019

Available Online:

10 June 2019

\section{Introduction}

AIDS caused by a retrovirus HIV has been globaly accepted as a pandemic problem with significant morbidity and mortality (World Health Organization WHO, 2014). It is estimated by United Nations Programme on HIV and AIDS (UNAIDS) that worldwide 
approximately 36.7 million people are already living with HIV (PLHIV) in 2016 which is likely to be increased with almost 1.8 million new infections each year (Global AIDS Update, 2017). The largest disease burden is shared by sub-Saharan Africa while India comes at the third position with approximately 20.89 lakh PLHIV (Global AIDS Update, 2017). Further, while approximately 2.23 milion people have died from AIDS, the mortality rate was noticed to be declined to 2.12 milion since 2007 (India HIV Estimations 2015 Technical Report, 2015). This steady decline in mortality rate is attributed to the introduction of highly active antiretroviral therapy (HAART) which exert improved clinical and laboratory outcomes in terms of fewer opportunistic infections and overall management of HIV/AIDS as a chronic illness (Moore and Chaisson, 1999; Fairal et al., 2008; Sow et al., 2007). Although, HAART has been established for its efficacy and significant benefits in reducing overall morbidity and mortality, it is also reported to exert unpleasant side effects and life-long medication thereby affecting cumulative health related quality of life (HRQoL) of PLHIV (Corless et al., 2005; Nicholas et al., 2005; Burgoyne and Tan, 2008).

HRQoLis defined as a multidimensional approach to address changes in overal health status including physical, mental and social functioning aspects either due to disease and/or treatment (Bonomi et al., 2000). In context of mental manifestations, neurocognitive disturbance and psychological problems such as depression and anxiety were extensively reported in PLHIV (Watkins and Treisman, 2015; NIMH, 2016). Neurocognitive disturbance in HIV is specifically defined as HIV-associated neurocognitive disorder (HAND) which includes $\mathrm{HIV}$-associated asymptomatic neurocognitive impairment (ANI), HIV- associated mild neurocognitive disorder (MND), and HIV-associated dementia (HAD) (Signh, 2012; Janssen et al., 1991; Ancuta et al., 2008). The pathophysiology behind neurological complications involves the infiltration of $\mathrm{HIV}$-infected immune cells through blood-brain barrier causing inflammation of the central nervous system by activating microglia and related pathways (Signh, 2012; Janssen et al., 1991; Ancuta et al., 2008). Further, psychological complications further enhances the HIV associated morbidity and mortality due to poor HRQoL, prognosis, response and adherence to HAART (Vivithanaporn et al., 2010; Hinkin et al., 2002; Tozzi et al., 2004; Heaton et al., 2004).

While a plethora of studies demonstrated the poor and improved HRQOL in PLHIV when compared with HIV free and HAART adhered HIV population respectively (Moore and Chaisson, 1999; Fairal et al., 2008; Sow et al., 2007; Muri et al., 2003; Wig et al., 2006; Pérez et al., 2009; Jelsma et al., 2005; Louwagie et al., 2007), evidences regarding the status of neurocognitive and psychological disorders in PLHIV at various stages of engagement in HIV care is not clear. Therefore, the present study aims to systematically review the evidences about the mental complications in PLHIV before and after HAART therapy.

\section{Materials and Methods}

The literature search was conducted for past ten years (i.e from 2008 to 2019) using the electronic databases like PubMed, Google scholar, Google. The search was conducted using the combination of keywords "AIDS" OR "HIV" AND "quality of life" OR "health related quality of life" AND "neurocognition", OR "cognitive deficit" OR "psychological complications" OR "mental health" OR "cognitive impairment" OR 
"depression" OR "anxiety" OR "dementia" AND "randomized controlled trials" OR "randomized clinical trials", OR "cohort study", OR "meta-analysis", OR "systemic review" OR "study". Only English language articles were searched and incorporated in the analysis.

Al randomized controlled trials irrespective of double blind, single blind or open, interventional studies, pilot studies, systematic reviews and meta-analysis were considered as eligible studies. Studies that compared the PLHIV with normal control, with or without therapy were selected although it has been ensured that all studies must have included mental health as primary or secondary parameter. Further studies conducted over adults above 18 years were only selected however studies conducted exclusively over one gender or specific age group for instance over aged population were excluded from the study design. Further, studies focused on specific pathologies in PLHIV in addition to mental complications or studies using interventions other than HAART were also not selected for this review in order to increase the study homogeneity.

\section{Results and Discussion}

A total of 27 studies were included in the current systematic review to assess the evidences about the neuropsychological health variables in PLHIV as represented in Table 1. Among the 27 studies, 11 studies recorded the neuropsychological variables as a component of HRQoL or QoL, remaining reports used the specific tools to assess depression, anxiety and HAND. In addition, the included studies involve 25 cross sectional studies and 2 reviews.

Among the 11 studies that investigated the mental health as a component of HRQoL, 10 studies showed overall poor mental performance including high depression and anxiety in PLHIV (Ledo et al., 2018; Nyongesa et al., 2018; Emuren et al., 2017; Thomas et al., 2017; Deshmukh et al., 2017; Maimaiti et al., 2017; Surur et al., 2017; Betancur et al., 2017; Akinboro et al., 2014; Briongos-Figuero, 2011; Campos et al., 2009). Further five studies unanimously reported depression as a major psychological disorder which overall negatively affect the QoL of PLHIV (Nyongesa et al., 2018; Emuren et al., 2017; Deshmukh et al., 2017; Maimaiti et al., 2017; Betancur et al., 2017; Briongos-Figuero, 2011). Similarly, in terms of the effect of HAART treatment in reduced predisposition to mental disorders, 5 studies reported positive effect. While studies conducted by Ledo et al., (2018) and Betancur et al., (2017) reported poor QoL including mental domains in HIV naïve and poor treatment adhering patients, cross sectional studies conducted by Akinboro et al., (2014), Campos et al., (2009) and Thomas et al., (2017) high lighted the significant effect of ART over modulation of psychological health and overall HRQoL scores specifically during the initial treatment period. On the contrary, remaining six studies demonstrated enhanced prevalence of depression and therefore poor QoL irrespective of ART administration. These studies additionally reported the significant association between female gender, smoking, and low CD4 count and worsened neuropsychological health and overall HRQoL.

HIV associated neurocognitive disorder (HAND) was studied by 8 studies either alone or as mixed diagnosis out of which 7 studies reported mild to severe HAND when compared either with healthy control or within HIV positive patients (Kumar et al., 2019; Yusuf et al., 2017; Balaini et al., 2017; Estiasari et al., 2015; Habib et al., 2013; Achappa et al., 2013; Wang et al., 2013). 
Only a single study conducted by Nyongesa $e t$ al., (2018) reported no significant effect of HIV over neurocognitive skills. Out of 8 studies, 6 studies included patients on HAART for different duration and majorly reported no response. While Balaini et al., (2017) and Nyongesa et al., (2018) found no association between HAND and cART regimen, studies conducted by Yusuf et al., (2017), Achappa et al., (2013), Wang et al., (2013) found mild to severe HAND prevalence irrespective of HAART administration.

The positive effect of long term administration of ART over HAND was recorded by Kumar et al., (2019) whereas study performed by Estiasari et al., (2015) reported poor cognitive performance and high Prevalence rate in absence of HAART treatment. In case of HAND major factors that were found to be associated with poor cognitive performance were long duration of HIV diagnosis, low CD4 count, low educational status, severity of illness, psychiatric diseases and substance use, anemia, low body mass index, increasing age, and female gender.

A total of 6 studies assessed depression and anxiety in PLHIV where 5 studies recorded high prevalence (Adeoti et al., 2018; Ramachandra and Badiger, 2018; Hafeez T, 2018; Betancur et al., 2017; Tesfaw et al., 2016) and one showed no significant occurrence (Gauiran et al., 2018).

All these studies included the patients on ART for variable duration, hence higher prevalence of depression and anxiety in these patients indicate no significant effect of treatment. Major correlates demonstrated by these studies include female gender, age, smoking, homosexuality, unprotected sex, unemployment, low CD4 count, non- disclosure of HIV status, perceived HIV stigma, poor social support, HIV stage I, poor medication adherence, divorce, and comorbid TB illness. Few number of studies $(\mathrm{n}=4)$ has assessed anxiety alone among PLHIV in which 3 were cross sectional studies and one was a review (Brandt et al., 2017; Mirghani and Elbadawi, 2017; Shukla et al., 2016; Belete et al., 2014).

Overall, the three cross sectional studies demonstrated low to high anxiety rate with variable severity irrespective of ART and interestingly study conducted by Belete et al., 2014 reported higher anxiety prevalence in ART receiving patients when compared with HIV naïve patients.

Similarly, review did by Brandt et al., 2017 demonstrated consistent relationship between increased anxiety prevalence and HIV medication non-adherence, substance use behavior, poor QoL, and suicidal tendency in PLHIV. Further, increased anxiety prevalence was associated with poor educational status, single marital status, perceived treatment side-effects, female gender, and perceived stigma about their HIV status.

The present study evaluated the evidences about the prevalence of common neuropsychological health disorders viz. HAND, depression, and anxiety among PLHIV. Further, the review also focused on the impact of HAART in decreasing the predisposition of mental disorders in PLHIV, if any.

Overall based upon the included studies it is evident that the enhanced coexistence of depression, anxiety and HAND with AIDS/HIV is common worldwide. This coexistence of mental disorders with HIV/AIDS can be understood in terms of pathophysiology mechanisms as well as social factors. 
Table.1 Summary of studies included to assess the mental health in HIV patients

\begin{tabular}{|c|c|c|c|c|c|}
\hline Author, Year & $\begin{array}{l}\text { Study type and } \\
\text { characteristics }\end{array}$ & Intervention & Compare at & Variables & Outcomes \\
\hline $\begin{array}{l}\text { Kumar et al., } \\
2019\end{array}$ & $\begin{array}{l}\text { Clinical study } \\
\mathrm{N}=200 \mathrm{HIV} \\
\text { patients } \\
(\mathrm{M}=130, \mathrm{~F}=70) \\
\mathrm{N}=200 \text { control }\end{array}$ & $\begin{array}{l}\mathrm{N}=182 \mathrm{on} \\
\text { HAART }\end{array}$ & $\begin{array}{l}\text { Healthy } \\
\text { control }\end{array}$ & -HAND & $\begin{array}{l}\text {-One-fourth of all HIV } \\
\text { patients had HAND. } \\
\text {-HigherCD4 Counts and } \\
\text { a Greater duration Of } \\
\text { ART decreases HAND } \\
\text { predisposition. }\end{array}$ \\
\hline $\begin{array}{l}\text { Ledo et al., } \\
2018\end{array}$ & $\begin{array}{l}\text { Cross sectional } \\
\text { study } \\
\mathrm{N}=104(\mathrm{M}=79 ; \\
\mathrm{F}=25)\end{array}$ & $\begin{array}{l}\text { No } \\
\text { intervention }\end{array}$ & $\begin{array}{l}\text { Within the } \\
\text { group }\end{array}$ & -HRQoL & $\begin{array}{l}\text {-Lower HRQoL in } \\
\text { Female patients } \\
\text { Specifically for Mental } \\
\text { Component } \\
\text { Summaries(MCS) of } \\
\text { HRQoL. } \\
\text {-Female gender and } \\
\text { smoking as Predictors } \\
\text { for MCS. }\end{array}$ \\
\hline $\begin{array}{l}\text { Nyongesa } \\
\text { et al., } 2018\end{array}$ & $\begin{array}{l}\text { A descriptive } \\
\text { cross-sectional } \\
\text { study. } \\
\mathrm{N}=167 \\
(\mathrm{M}=48, \mathrm{~F}=119)\end{array}$ & $\begin{array}{l}\text { HAART } \\
(\mathrm{n}=84)\end{array}$ & $\begin{array}{l}\text { Healthy } \\
\text { community } \\
\text { control } \\
(\mathrm{n}=83)\end{array}$ & $\begin{array}{l}\text {-Non-verbal } \\
\text { Intelligence, } \\
\text { verbal working } \\
\text { memory and } \\
\text { executive } \\
\text { Functioning. } \\
\text { - Depression } \\
\text {-QoL }\end{array}$ & $\begin{array}{l}\text {-No major effect of HIV } \\
\text { infection on } \\
\text { neurocognitive tests. } \\
\text {-Increased depression } \\
\text { scores in HIV patients. } \\
\text {-No correlation between } \\
\text { neurocognitive scores } \\
\text { and QoL but strong } \\
\text { association between } \\
\text { depression and QoL. }\end{array}$ \\
\hline $\begin{array}{l}\text { Adeoti et al., } \\
2018\end{array}$ & $\begin{array}{l}\text { A cross- } \\
\text { Sectional study } \\
\mathrm{N}=753(\mathrm{M}=165, \\
\mathrm{F}=588) \mathrm{HIV} \\
\text { positive }=424, \\
\text { HIV negative } \\
=329)\end{array}$ & $\begin{array}{l}\text { ART for } 6 \\
\text { months }\end{array}$ & Healthy control & $\begin{array}{l}\text {-Anxiety and } \\
\text { depression }\end{array}$ & $\begin{array}{l}\text {-High prevalence of } \\
\text { depression and Anxiety } \\
\text { disorders And their co- } \\
\text { morbid occurrence. }\end{array}$ \\
\hline $\begin{array}{l}\text { Ramachandra } \\
\text { and Badiger, } \\
2018\end{array}$ & $\begin{array}{l}\text { A cross Sectional } \\
\text { study } \\
N=169(M=110, \\
F=59)\end{array}$ & ART & $\begin{array}{l}\text { Within the } \\
\text { group }\end{array}$ & $\begin{array}{l}\text {-Anxiety and } \\
\text { depression }\end{array}$ & $\begin{array}{l}\text { More frequent in } \\
\text { females -Females were } \\
\text { Prone to anxiety } \\
(62.1 \%) \text { and depression } \\
(59.4 \%) \text { - Association } \\
\text { between disease } \\
\text { diagnosis age and } \\
\text { depression. }\end{array}$ \\
\hline
\end{tabular}




\begin{tabular}{|c|c|c|c|c|c|}
\hline $\begin{array}{l}\text { Hafeez, } \\
2018\end{array}$ & $\begin{array}{l}\text { Comparative } \\
\text { study } \\
\mathrm{N}=168(\mathrm{M}=120, \\
\mathrm{F}=48)\end{array}$ & $\begin{array}{l}\mathrm{N}=85 \mathrm{on} \\
\mathrm{HAART} \text { and } \\
\mathrm{N}=83 \\
\text { without } \\
\text { treatment }\end{array}$ & HIV naive & $\begin{array}{l}\text {-Anxiety and } \\
\text { depression }\end{array}$ & $\begin{array}{l}\text {-Both depression and } \\
\text { anxiety were Higher } \\
\text { among HIV/AIDS } \\
\text { patients who are not on } \\
\text { treatment. } \\
\text {-Depression is Primarily } \\
\text { higher Irrespective of } \\
\text { treatment. } \\
\text {-Females and Unmarried } \\
\text { male With CD4<500 are } \\
\text { more prone for } \\
\text { depression and anxiety. }\end{array}$ \\
\hline $\begin{array}{l}\text { Gauiran et al., } \\
2018\end{array}$ & $\begin{array}{l}\text { Cross-sectional } \\
\text { Analytic study } \\
\mathrm{N}=417(\mathrm{M}=408, \\
\mathrm{F}=9)\end{array}$ & $\begin{array}{l}\mathrm{N}=362 \mathrm{on} \\
\text { ART }\end{array}$ & $\begin{array}{l}\text { With in the } \\
\text { group }\end{array}$ & $\begin{array}{l}\text {-Anxiety } \\
\text { and } \\
\text { depression }\end{array}$ & $\begin{array}{l}\text {-Low prevalence Rate of } \\
\text { anxiety and/or } \\
\text { depression }(10.1 \%) \text {, - } \\
\text { Significant associates } \\
\text { were cigarete smoking, } \\
\text { homosexuality, } \\
\text { Unprotected sex, } \\
\text { unemployment, } \\
\text { Female sex and non- } \\
\text { disclosure of status }\end{array}$ \\
\hline $\begin{array}{l}\text { Emuren et al., } \\
2017\end{array}$ & $\begin{array}{l}\text { Cohort } \\
N=1668 \\
(M=1552, \\
F=116)\end{array}$ & $\begin{array}{l}\text { HAART } \\
(\mathrm{n}=1257)\end{array}$ & $\begin{array}{l}\text { Within } \\
\text { HAART } \\
\text { treatment }\end{array}$ & -HRQoL & $\begin{array}{l}\text {-Depression Accounted } \\
\text { for Over } 60 \% \text { of the } \\
\text { psychological } \\
\text { Comorbidity and Most } \\
\text { predictive Factor of } \\
\text { HRQOL }\end{array}$ \\
\hline $\begin{array}{l}\text { Thomas et al., } \\
2017\end{array}$ & $\begin{array}{l}\text { Large cross- } \\
\text { Sectional survey } \\
\text { Done in Zambia } \\
\text { and South Africa. } \\
\text { Zambia: } \\
19733 \\
\text { respondents } \\
\\
(\mathrm{M}=5428 ; \mathrm{F}= \\
14305)(\mathrm{HIV} \\
\text { positive=4128) } \\
\text { South Africa: } \\
18612 \\
\text { respondents } \\
(\mathrm{M}=5816 ; \mathrm{F}= \\
12796)(\mathrm{HIV} \\
\text { positive4012). }\end{array}$ & $\begin{array}{l}\text { In Zambia: } \\
\mathrm{N}=1585 \text { on } \\
\text { HAART } \\
\text { in South } \\
\text { Africa: N= } \\
\text { 1236on } \\
\text { HAART }\end{array}$ & $\begin{array}{l}\text { HIV } \\
\text { Negative and } \\
\text { HIV naive }\end{array}$ & HRQoL & $\begin{array}{l}\text {-No significant } \\
\text { difference in overall } \\
\text { HRQoL scores between } \\
\text { HIV positive and } \\
\text { negative individuals } \\
\text { where ART has been } \\
\text { used for more than } 5 \\
\text { years. } \\
\text {-Improved HRQoL } \\
\text { scores between HIV- } \\
\text { positive and negative } \\
\text { Individuals who had } \\
\text { initiated ART less than } \\
5 \text { years previously }\end{array}$ \\
\hline Deshmukh & A & ART & Within the & -Depression, & -Depression was seen in \\
\hline
\end{tabular}




\begin{tabular}{|c|c|c|c|c|c|}
\hline et al., 2017 & $\begin{array}{l}\text { cross- sectional } \\
\text { study } N=754 \\
(M=460 \\
F=294)\end{array}$ & & group & $\begin{array}{l}\text { Anxiety and } \\
\text { stress } \\
\text { scale- } 21 \\
\text {-QOL }\end{array}$ & $\begin{array}{l}50 \% \text { of the patients } \\
\text {-Depression more } \\
\text { prevalent in Females - } \\
\text { Depressed } \\
\text { Patients have Overall } \\
\text { lower QOL. }\end{array}$ \\
\hline $\begin{array}{l}\text { Maimaiti } \\
\text { et al., } 2017\end{array}$ & $\begin{array}{l}\text { Consecutive } \\
\text { Case series } \\
\mathrm{N}=679(\mathrm{M}=411, \\
\mathrm{F}=268)\end{array}$ & ART & $\begin{array}{l}\text { Within the } \\
\text { group }\end{array}$ & -HRQoL & $\begin{array}{l}-69 \% \text { HIV cases showed } \\
\text { depressive } \\
\text { symptoms. }\end{array}$ \\
\hline $\begin{array}{l}\text { Surur et al., } \\
2017\end{array}$ & $\begin{array}{l}\text { Cross-sectional } \\
\text { study } \\
\mathrm{N}=400(\mathrm{M}=181, \\
\mathrm{F}=219)\end{array}$ & HAART & $\begin{array}{l}\text { Within the } \\
\text { group }\end{array}$ & -HRQoL & $\begin{array}{l}\text {-Alldomains of HRQoL } \\
\text { were Found to be } \\
\text { moderate } \\
\text { How ever the } \\
\text { psychological Health } \\
\text { was found to be lower } \\
\text { than remaining domains. }\end{array}$ \\
\hline $\begin{array}{l}\text { Betancur } \\
\text { et al., } 2017\end{array}$ & $\begin{array}{l}\text { Cross-sectional } \\
\text { study. } \\
\mathrm{N}=47 \\
(\mathrm{M}=14, \mathrm{~F}=33)\end{array}$ & ART & $\begin{array}{l}\text { Within the } \\
\text { group }\end{array}$ & $\begin{array}{l}\text {-Socio- } \\
\text { demographic } \\
\text { variables, } \\
\text { depression and } \\
\text { anxiety in poor } \\
\text { adherence to } \\
\text { HAART HIV } \\
\text { patients }\end{array}$ & $\begin{array}{l}-59.5 \% \text { participants } \\
\text { presented moderate to } \\
\text { Severe depressive } \\
\text { symptoms. } \\
\text {-Poor QoLinnon- } \\
\text { adhering patients with } \\
\text { mental health as the } \\
\text { most affected variable. } \\
\text {-Females constituted the } \\
\text { Higher proportion of } \\
\text { non-adherent patients. }\end{array}$ \\
\hline $\begin{array}{l}\text { Brandt et al., } \\
2017\end{array}$ & Review & NA & NA & -Anxiety & $\begin{array}{l}(\mathrm{n}=1) \text {, and Positive } \\
\text { relation }(\mathrm{n}=1) \text {. } \\
\text {-Anxiety and HIV } \\
\text { medication } \\
\text { Adherence ( } \mathrm{n}=13) \\
\text { =positive relation } \\
\text { between Increased } \\
\text { anxiety and HIV } \\
\text { Medication non- } \\
\text { Adherence ( } \mathrm{n}=10) \text {. } \\
\text {-Consistent significant } \\
\text { relation between anxiety } \\
\text { symptoms and substance } \\
\text { use behavior }(\mathrm{n}=4) \\
- \text {-Anxiety and Sexual } \\
\text { risk( } \mathrm{n}=9)=\mathrm{positive} \\
\text { relationship }(\mathrm{n}=4) \text {, } \\
\text { No relations }(\mathrm{n}=2) \text {, and }\end{array}$ \\
\hline
\end{tabular}




\begin{tabular}{|c|c|c|c|c|c|}
\hline & & & & & $\begin{array}{l}\text { negative relations } \\
(\mathrm{n}=2) \text {. } \\
\text {-Consistent relationship } \\
\text { between anxiety and } \\
\text { lower QoL. } \\
\text {-Consistent relationship } \\
\text { between anxiety and } \\
\text { suicidal thoughts, } \\
\text { Behaviors and history } \\
(\mathrm{n}=4)\end{array}$ \\
\hline $\begin{array}{l}\text { Mirghani } \\
\text { and } \\
\text { Elbadawi, } \\
2017\end{array}$ & $\begin{array}{l}\text { A cross-sectional } \\
\text { Analytic study } \\
\mathrm{N}=352(\mathrm{M}=220 \\
\mathrm{F}=132)\end{array}$ & ART & $\begin{array}{l}\text { Within the } \\
\text { group }\end{array}$ & -Anxiety & $\begin{array}{l}\text {-High anxiety rate in } \\
\text { HIV/AIDS } \\
\text { patients } \\
\text { Especially among } \\
\text { illiterate, widowed/ } \\
\text { divorced }\end{array}$ \\
\hline $\begin{array}{l}\text { Yusuf et al., } \\
2017\end{array}$ & $\begin{array}{l}\text { Cross-sectional } \\
\text { study } \\
\mathrm{N}=418(\mathrm{M}=93, \\
\mathrm{F}=325)\end{array}$ & ART & $\begin{array}{l}\text { Within the } \\
\text { group }\end{array}$ & -HAND & $\begin{array}{l}\text {-Prevalence rate of } 19 \% \text {. } \\
\text {-Major correlates were } \\
\text { duration of HIV } \\
\text { diagnosis, } \\
\text { Low CD4 count and } \\
\text { high detectable viral } \\
\text { load during ART, } \\
\text { Low educational status, } \\
\text { and Severity of illness. }\end{array}$ \\
\hline $\begin{array}{l}\text { Balaini et al., } \\
2017\end{array}$ & $\begin{array}{l}\begin{array}{l}\text { Prospective } \\
\text { observational } \\
\text { study } \\
\mathrm{N}=41(\mathrm{M}=25, \\
\mathrm{F}=16)\end{array}\end{array}$ & HAART & $\begin{array}{l}\text { Within the } \\
\text { group }\end{array}$ & -HAND & $\begin{array}{l}\text {-HAND is Common } \\
\text { among HIV patients } \\
\text { with asymptomatic } \\
\text { neurocognitive } \\
\text { Impairment as the Most } \\
\text { prevalent type. } \\
\text {-No association } \\
\text { Between HAND and } \\
\text { cART regimen. }\end{array}$ \\
\hline $\begin{array}{l}\text { Shukla et al., } \\
2016\end{array}$ & $\begin{array}{l}\text { Hospital- based } \\
\text { cross-sectional } \\
\text { study } \\
\mathrm{N}=170(\mathrm{M}=110, \\
\mathrm{F}=60)\end{array}$ & ART & $\begin{array}{l}\text { Within the } \\
\text { group }\end{array}$ & -Anxiety & $\begin{array}{l}\text { - All patients showed } \\
\text { anxiety with } 92.1 \% \\
\text { showed mild anxiety } \\
\text { while remaining } \\
\text { moderate to severe } \\
\text { anxiety symptoms. } \\
\text {-Anxiety severity was } \\
\text { associated with } \\
\text { educational status, } \\
\text { perceived side-effects } \\
\text { during last one month } \\
\text { and duration of }\end{array}$ \\
\hline
\end{tabular}




\begin{tabular}{|c|c|c|c|c|c|}
\hline & & & & & treatment. \\
\hline \multirow[t]{2}{*}{$\begin{array}{l}\text { Tesfaw et al., } \\
2016\end{array}$} & $\begin{array}{l}\text { Institution } \\
\text { Based cross- } \\
\text { Sectional study. } \\
N=417(M=166, \\
F=251)\end{array}$ & ART & $\begin{array}{l}\text { Within the } \\
\text { group }\end{array}$ & $\begin{array}{l}\text {-Anxiety } \\
\text { and } \\
\text { depression }\end{array}$ & $\begin{array}{l}-41.2 \% \text { had Depression } \\
\text { and } 32.4 \% \text { had anxiety } \\
\text { while } 24.5 \% \text { showed } \\
\text { co- morbid Depression } \\
\text { and anxiety. }\end{array}$ \\
\hline & & & & & $\begin{array}{l}\text {-Major correlates For } \\
\text { depression Were } \\
\text { perceived HIVstigma, } \\
\text { poor Social support, } \\
\text { HIV stage I and Poor } \\
\text { medication adherence. } \\
\text {-Major correlates For } \\
\text { anxiety were Female } \\
\text { gender, divorce, co- } \\
\text { morbid TB illness and } \\
\text { Perceived HIV stigma. }\end{array}$ \\
\hline $\begin{array}{l}\text { Estiasari } \\
\text { et al., } 2015\end{array}$ & $\begin{array}{l}\text { Cross-sectional } \\
\text { study } \\
\mathrm{N}=82(\mathrm{M}=56, \mathrm{~F}= \\
26)\end{array}$ & $\begin{array}{l}\text { No } \\
\text { treatment }\end{array}$ & $\begin{array}{l}\text { Healthy } \\
\text { control }\end{array}$ & -HAND & $\begin{array}{l}\text {-Poor cognitive } \\
\text { Performance of HIV } \\
\text { subjects in Comparison } \\
\text { to Healthy controls. } \\
\text {-Prevalence rate Of } 51 \% \\
\text { in HIV Naïve patients. }\end{array}$ \\
\hline $\begin{array}{l}\text { Belete et al., } \\
2014\end{array}$ & $\begin{array}{l}\text { Institute based } \\
\text { cross-sectional } \\
\text { study } \\
N=436(M=174, \\
F=262)\end{array}$ & $\begin{array}{l}72 \% \text { on } \\
\text { HAART }\end{array}$ & $\begin{array}{l}\text { HIV naïve } \\
\text { patients }\end{array}$ & -Anxiety & $\begin{array}{l}\text {-Anxiety Prevalence rate } \\
\text { Was } 22.2 \% \text {. } \\
\text {-Major anxiety } \\
\text { Correlates were Female } \\
\text { gender, Divorced and } \\
\text { Perceived stigma About } \\
\text { their HIV status. } \\
\text {-Patients on ART } \\
\text { Showed } 2.7 \text { times More } \\
\text { prevalence Of anxiety in } \\
\text { Comparison to HIV } \\
\text { naïve patients }\end{array}$ \\
\hline
\end{tabular}




\begin{tabular}{|c|c|c|c|c|c|}
\hline $\begin{array}{l}\text { Akinboro } \\
\text { et al., } 2014\end{array}$ & $\begin{array}{l}\text { Cross-sectional } \\
\text { study } \\
N=491(M=144, \\
F=347)\end{array}$ & $\begin{array}{l}\mathrm{N}=393 \text { on } \\
\text { HAART }\end{array}$ & $\begin{array}{l}\text { Within the } \\
\text { group }\end{array}$ & WHO-QoL & $\begin{array}{l}\text {-Participants with CD4 } \\
\text { count } \geq 350 \text { cels } / \mathrm{mm}^{3} \text { had } \\
\text { better QOL scores in the } \\
\text { physical, Psychological } \\
\text { and Level of } \\
\text { independence domains. } \\
\text {-Subjects on } \\
\text { antiretroviral } \\
\text { therapy(ART) reported } \\
\text { Significantly Better } \\
\text { QOL in the physical, } \\
\text { psychological, } \\
\text { level of Independence } \\
\text { and spirituality } \\
\text { domains. }\end{array}$ \\
\hline $\begin{array}{l}\text { Habib et al., } \\
2013\end{array}$ & $\begin{array}{l}\text { Random effects } \\
\text { meta-analysis } \\
\text { Of prospective } \\
\text { studies }\end{array}$ & NA & NA & -HAND & $\begin{array}{l}\text {-HIV associated } \\
\text { With NCI. } \\
\text {-ART lowers NCI } \\
\text { By } 63 \% \text { and } 77 \% \\
\text { When compared } \\
\text { To HIV naïve } \\
\text { Patients and } 6\end{array}$ \\
\hline $\begin{array}{l}\text { Achappa } \\
\text { et al., } 2013\end{array}$ & $\begin{array}{l}\text { Crosssectional } \\
\text { study } \\
\mathrm{N}=101(\mathrm{M}=69 \\
\mathrm{F}=32)\end{array}$ & $\begin{array}{l}\mathrm{N}=88 \mathrm{on} \\
\text { HAART }\end{array}$ & $\begin{array}{l}\text { Within the } \\
\text { group }\end{array}$ & -HAND & $\begin{array}{l}\text { Months treatment } \\
\text { respectively. } \\
\text {-Psychiatric Diseases } \\
\text { and Substance use } \\
\text { Further enhances NCI } \\
\text { prevalence. } \\
\text {-91 out of } 101 \text { Patients } \\
\text { had HAND. } \\
\text {-Risk factors were Low } \\
\text { CD4 cel counts, anemia, } \\
\text { Low bodymass index, } \\
\text { increasing age, and } \\
\text { female gender. }\end{array}$ \\
\hline $\begin{array}{l}\text { Wang et al., } \\
2013\end{array}$ & $\begin{array}{l}\text { Cross-sectional } \\
\text { survey } \\
N=309(\mathrm{M}=272, \\
\mathrm{F}=37)\end{array}$ & $\begin{array}{l}\mathrm{N}=236 \mathrm{on} \\
\text { HAART }\end{array}$ & $\begin{array}{l}\text { Within the } \\
\text { group }\end{array}$ & -HAND & $\begin{array}{l}\text {-Higher Prevalence rate } \\
\text { of HAND in HIV- } \\
\text { Infected patients With a } \\
\text { baseline CD4 count } \\
\leq 350 \text { cels/ } \mu \text { L. } \\
\text {-Major correlates Were } \\
\text { oldage, Female gender, } \\
\text { Low level of education, } \\
\text { and a Longer period of } \\
\text { EFV use in HAART }\end{array}$ \\
\hline
\end{tabular}




\begin{tabular}{|c|c|c|c|c|c|}
\hline & & & & & $\begin{array}{l}\text { regimens } \\
\text {-Depression }\end{array}$ \\
\hline $\begin{array}{l}\text { Briongos- } \\
\text { Figuero, } 2011\end{array}$ & $\begin{array}{l}\text { cross-sectional } \\
\text { study } \\
\mathrm{N}=150(\mathrm{M}=112 \\
\mathrm{F}=38)\end{array}$ & ART & $\begin{array}{l}\text { Within the } \\
\text { group }\end{array}$ & $\begin{array}{l}\text {-Depression } \\
\text {-HRQoL }\end{array}$ & $\begin{array}{l}\text { Significantly and } \\
\text { negatively } \\
\text { Affected alHRQL } \\
\text { Domains including } \\
\text { Mental Health } \\
\text { Summary(MHS) }\end{array}$ \\
\hline $\begin{array}{l}\text { Campos et al., } \\
2009\end{array}$ & $\begin{array}{l}\text { A prospective } \\
\text { adherence } \\
\text { study. } \\
\mathrm{N}=262\end{array}$ & ART & $\begin{array}{l}\text { Baseline } \\
\text { Values without } \\
\text { ART treatment }\end{array}$ & $\begin{array}{l}\text {-Quality of } \\
\text { life } \\
\text {-Anxiety } \\
\text { And } \\
\text { depression } \\
\text { symptoms }\end{array}$ & $\begin{array}{l}\text {-Improved QoL } \\
\text { After four months } \\
\text { Of ART } \\
\text {-Lackofanxiety } \\
\text { And depression } \\
\text { symptoms } \\
\text { Associated with } \\
\text { goodQoL. }\end{array}$ \\
\hline
\end{tabular}

In terms of biological mechanisms, occurrence of HAND in HIV patients was attributed to the CNS viral reservoir and neuroinflammatory pathways (Cysique et al., 2015). It is hypothesized that both CNS and peripheral monocytes and macrophages serve as HIV reservoirs due to longer lifespan and rescue mechanisms from HIV infection or immune surveillance (Zhu et al., 2002; Bacchus et al., 2013; Campbel et al., 2014; He et al., 1997; Lavi et al., 1997). Further, due to the chronic nature of disease, a constant low-grade immune activation and inflammation persists which act as a potential contributor to HAND (Freund et al., 2010; Cysique et al., 2013). Similarly, mood disorders particularly depression and anxiety have been associated with cortical and subcortical regions in HIV negative patients (Drevets and Neuroimaging, 2000; Sheline, 2000). However, their direct role in increased psychological vulnerability among HIV positive patients is stil not clear. In addition, hypothalamus-pituitary-thyroid (HPT) and hypothalamic-pituitary-adrenal (HPA) axis dysfunction has also been established in mood disturbances which may play significant role in the pathogenesis of depression and anxiety in PLHIV (Langford et al., 2011). Over activation of HPA axis may further increase the HIV disease progression through enhanced cortisol secretion which in turn can alter T-lymphocyte cytokine production, destruction of CD4 lymphocytes and therefore stimulated HIV replication (Sadock and Sadock, 2005). Moreover, elevated cortisols ecretion proportionaly influenced the norepinephrine synthesis which further stimulates HIV replication (Cole et al., 1998). Elevated tryptophan degradation which serves as a serotonin precursor is also illustrated in PLHIV. Increased tryptophan degradation further reduces immune activation as wel as reduced serotonin synthesis together causing enhanced HIV disease and psychological disorders progression (Schroecksnadel et al., 2008).

Neuropsychological disorders in PLHIV were largely related with stressful life events and diminished social support (Leserman et al., 2002; Ironson et al., 2005). The present review also observed that major correlates for enhanced neuropsychological disorders involve social factors such as being female, HIV stigma, low education and income status, societal isolation, poor family support, smoking and substance use. Larger 
vulnerability of females towards mental disorders can be attributed to factors such as increased exposure to acute life events, lower social status and network, and financial problem (NACA, 2012). HIV stigma serves as one of the leading factor in increased preponderance of depression and anxiety. Stigma results in enhanced fatigue levels, isolation, loneliness and feling of worthlessness (Rodkjaer et al., 2010; Bhate and Munjal, 2014; Berhe and Bayray, 2013). Similarly, social relationship domain not only help in preventing mental disorders but also significantly affect overall QoLinPLHIV as it provides safety, security and financial support. Smoking and substance use bidirectionaly indicate status of mental problems as wel as disease progression and therefore interventions to stop them are inherent part of HIV management (Chang et al., 2017; Ruggles et al., 2017).

Based upon the present review, the role of HAART in reducing the prevalence of neuropsychological disorders with disease progression is largely meager. This poor effect of HAART can be attributed to irreversible CNS damage occurred during the early disease course before the start of intervention, sustained neuroinflammation, viral replication and load in CNS while on HAART (Becker et al., 2011; Dahl et al., 2014). In-addition, an observational study also demonstrated the neurotoxic effect of HAART specifically by the antibiotics used as first line of treatment (Bacchus et al., 2013). Patients CD4 count also serve as a prognostic factor for HAART response against mental disorders as a low or nadir CD4 count indicate advanced disease state and immune damage.

Overal, based upon the current evidences while it can be concluded that the prevalence of neuropsychological disorders increased with HIV disease which negatively influenced the cumulative QoL of PLHIV and HAART is not sufficient on its own to manage them, several important caveats has been noticed in the available literature. First more than 50\% studies assessed mental complications as a component of HRQoL thereby considering it as a secondary objective. Second, methodologies and study design used to assess mental disorders varied significantly which can largely impact the study outcomes. Third, studies examining the impact of neuropsychological disorders on disease progression have not been addressed due to the inclusion of subjective questioners and lack of analysis of immune system biomarkers. Fourth, although most of the studies used patients on HAART the treatment duration, baseline disease aswelas mental status and folowup time was not mentioned. Therefore it is recommended that baseline assessment of HRQoL, immune markers, and neuropsychological disorders may serve as beter treatment strategy with improved outcomes. Further, considering the serious repercussions of mental disorders on HRQoL, it would be beneficial to incorporate additional treatment regimen for the min addition to HAART from the beginning.

\section{References}

Abdrrahman S, Fitsum T, Wondwessen W, et al., Health related quality of life of HIV/AIDS patients on highly active anti-retroviral therapy at a university referral hospital in Ethiopia. BMC Health Services Research. 2017; 17: 737.

Abdulkareem Y, Abdulaziz H, Aisha M, et al., Prevalence of HIV-Associated Neurocognitive Disorder (HAND) among Patients Atending a Tertiary Health Facility in Northern Nigeria. J Int Assoc Provid AIDS Care. 2017; 16(1): 48-55.

Abdulrazaq H, Ahmad Y, Lukman O, et al., 
Neurocognitive impairment in HIV-1infected adults in Sub-Saharan Africa: a systematic review and meta-analysis. International Journal of Infectious Diseases. 2013; 17: e820-e831.

Adeolu A, Suliat A, Peter O, et al., Quality of life of Nigerians living with human immunodeficiency virus. Pan African Medical Journal. 2014; 18: 234.

Adeoti AO, Dada MU, Fadare JO. Prevalence of Depression and Anxiety Disorders in People Living with HIV/AIDS in a Tertiary Hospital in South Western Nigeria. Med Rep Case Stud. 2018; 3: 150.

Amsalu B, Gashaw A, Minale T, et al., Prevalence of Anxiety and Associated Factors among People Living with HIV/AIDS at Debretabor General Hospital Anti Retro Viral Clinic Debretabor, Amhara, Ethiopia, 2014. American Journal of Psychiatry and Neuroscience. 2014; 2(6): 109-114.

Ana L, Indira P, Liliane L, Mansueto N, and Carlos B. Association Between HealthRelated Quality of Life and Physical Functioning in Antiretroviral-Naïve HIV-Infected Patients. The Open AIDS Journal. 2018; 12: 117-125.

Ancuta P, Kamat A, Kunstman KJ, et al., Microbial translocation is associated with increased monocyte activation and dementia in AIDS patients. PLoSOne 2008; 3: e2516.

Antinori A, Arendt G, Becker JT, et al., Updated research nosology of HIV associated neurocognitive disorder. Neurology. 2007; 69(18): 1789-1799.

Bacchus C, Cheret A, Avet and-Fenoël V, et al.: A single HIV-1 cluster and a skewed immune homeostasis drive the early spread of HIV among resting CD4+ cell subsets within one month post-infection. PLoSOne. 2013; 8(5): e64219.

Becker T, Sanders J, Madsen SK, et al.,
Subcortical brain atrophy persists even in HAART -regulated HIV disease. Brain Imaging Behav. 2011; 5(2): 7785.

Berhe H, Bayray A. Prevalence of depression and associated factors among people living with HIV/AIDSI in Tigray, Ethiopia. North Ethiopia: A crosssectional hospital based study. IJPSR. 2013; 4(2): 765-75.

Bhate MS, Munjal S. Prevalence of depression in people living with HIV/AIDS undergoing ART and factors associated with it. J Clin Diagn Res. 2014; 8(10): WC01-4.

Bonomi AE, Patrick DL, Bushnel DM, Martin M. Validation of the United States' version of the World Health Organization Quality of Life (WHOQOL) instrument. J Clin Epidemiol. 2000 Jan; 53(1): 1-12.

Briongos-figuero L, Bachiler-luque $\mathrm{P}$, Palacios-martín $\mathrm{T}$, et al., Depression and health related quality of life among HIV-infected people. European Review for Medical and Pharmacological Sciences. 2011; 15: 855-862.

Burgoyne RW, Tan DH. Prolongation and quality of life for HIV-infected adults treated with highly active antiretroviral therapy (HAART): a balancing act. The Journal of antimicrobial chemotherapy. 2008; 61(3): 469 \pm 73 .

Campbel JH, Hearps AC, Martin GE, et al.: The importance of monocytes and macrophages in HIV pathogenesis, treatment, and cure. AIDS. 2014; 28(15): 2175-2187.

Chang L, Lim A, Lau E, Alicata D. Chronic tobacco-Smoking psychopathological symptoms, impulsivity and cognitive deficits in HIV infected individuals. J Neuroimmune Pharmacol. 2017; 12(3): $389-401$.

Charles B, Michael Z, Steven $\mathrm{P}$, et al., 
Anxiety symptoms and disorders among adults living with HIV and AIDS: A critical review and integrative synthesis of the empirical literature. Clin Psychol Rev. 2017; 51: 164-184.

Cole WS, Korin YD, Fahey J, Zack J. Norepinephrine accelerates HIV replication via protein kinase-A dependent effects on cytokine production. J. Immunol. 1998; 161: 610-616.

Corless IB, Kirksey KM, Kemppainen J, Nicholas PK, McGibbon C, Davis SM, et al., Lipodystrophy associated symptoms and medication adherence in HIV/AIDS. AIDS patient care and STDs. 2005; 19(9): 577 \pm 86 .

Cysique LA, Hey-Cunningham WJ, Dermody N, et al.: Peripheral blood mononuclear cells HIV DNA levels impact intermittently on neurocognition. PLoSOne. 2015; 10(4): e01204 88.

Cysique LA, Moffat K, Moore DM, et al.: HIV, vascular and aging injuries in the brain of clinically stable HIV-infected adults: A1HMRS Study. PLoS One. 2013; 8(4): e61738.

Dahl V, Peterson J, Fuchs D, et al., Low levels of HIV-1 RNA detected in the cerebrospinal fluid after upto 10 years of suppressive therapy are associated with local immune activation. AIDS. 2014; 28(15): 2251-2258.

Deonne G, Kenneth S, Jodor A, et al., Lourdes Rosanna E. DeGuzman. Measurement of Anxiety and Depression among HIV Patients seen in the Philippine General Hospital using the Hospital Anxiety and Depression Scale-Pilipino Version (HADS-P). Actamedica philippina. 2018; 52(1).

Deshmukh NN, Borkar AM, Deshmukh JS. Depression and its associated factors among people living with HIV/AIDS: Can it affect their quality of life? J Family Med Prim Care. 2017; 6: 549-53
Drevets WC. Neuroimaging studies of mood disorders. Biol. Psychiatry 2000; 48: 813-8 29.

Emuren L, Weles S, Evans AA, et al., (2017) Health-related quality of life among military HIV patients on antiretroviral therapy. PLoSONE 12(6): e0178953

Fairal LR, Bachmann MO, Louwagie GM, et al., Effectiveness of antiretroviral treatment in a South African program: A cohort study. Arch Intern Med. 2008 Jan 14; 168(1): 86-93.

Fairal LR, Bachmann MO, Louwagie GM, et al., Effectiveness of antiretroviral treatment in a South African program: A cohort study. Arch Intern Med. 2008 Jan $14 ; 168(1)$ : 86-93.

Freund A, Orjalo AV, Desprez PY, et al.: Inflammatory networks during cellular senescence: causes and consequences. Trends Mol Med. 2010; 16(5): 238246.

Getachew T, Getinet A, Tadesse A, et al., Prevalence and correlates of depression and anxiety among patients with HIV on follow-up at Alert Hospital, Addis Ababa, Ethiopia. BMC Psychiatry. 2016; 16: 368.

Hafeez T. A Comparative Study of Depression and Anxiety in HIV/AIDS Patients registered at treatment center in Lahore Pakistan. J Med Res Biol Stud. 2018; 1: 106.

Heaton RK, Clifford DB, Franklin DR, et al., HIV-associated neurocognitive disorders persist in the era of potent antiretroviral therapy: CHARTER Study. Neurology 2010; 75: 2087-96.

Heaton RK, Marcote TD, Mindt MR, et al., The impact of HIV associated neuropsychological impairment on every day functioning. J Int Neuropsychol Soc 2004; 10: 317-31.

He J, Chen Y, Farzan M, et al.: CCR3 and CCR5 are co-receptors for HIV-1 infection of microglia. Nature. 1997; 
385(6617): 645-649.

Hinkin CH, Castelon SA, Durvasula RS, et al., Medication adherence among HIV+ adults: effects of cognitive dysfunction and regimen complexity. Neurology 2002; 59: 1944-50.

Hyder, O., Mirghani, Abdulateef S. Elbadawi. Anxiety among HIV/AIDS Sudanese patients: A cross sectional analytic study. Indian Journal of Basic and Applied Medical Research. 2017; 6(2): 615-622.

Ironson G, O'Cleirigh C, Fletcher MA et al., Psychosocial factors predict CD4 and viral load change in men and women with human immunodeficiency virus in the era of highly active antiretroviral treatment. Psychom. Med. 2005; 67: 1013-1021.

Isabel Ruiz Pérez, Antonio Olryde Labry Lima, Luis Sordodel Castilo, Jesús Rodríguez Baño, Miguel Ángel López Ruz, Alfonsodel Arco Jimenez. No differences in quality of life between men and women undergoing HIV antiretroviral treatment: Impact of demographic, clinical and psychosocial factors. AIDS Care. 2009; 21(8): 943952.

Janssen RS, Cornblath DR, Epstien LG. Nomenclature and research case definition for neurologic manifestation of human immunodeficiency virus-type(HIV1) infection. Report of a working group of the American Academy of Neurology AIDSTask Force. Neurology. 1991; 41(6): 778-785.

Jelsma J, Maclean E, Hughes J, Tinise X, Darder M. An investigation into the health-related quality of life of individuals living with HIV who are receiving HAART. AIDSCare. 2005; 17(2): 579-588.

Kavya R, Sanjeev B. Depression and anxiety among people living with HIV in a coastal city of Karnataka. Int $\mathbf{J}$
Community Med Public Health. 2018; 5(7): 2931-2934

Langford D, Baron D, Joy J, Del Vale L, Shack J. Contributions of HIV infection in the hypothalamus and substance abuse/use to HPT dysregulation. Psychoneuro endocrinology 2011; 36: 710-719.

Lavi E, Strizki JM, Ulrich AM, et al.: CXCR4(Fusin), a co-receptor for the type 1 human immunodeficiency virus (HIV1 ), is expressed in the human brain in a variety of cell types, including microglia and neurons. Am J Pathol. 1997; 151(4): 1035-1042.

Leserman J, Petito JM, GuH et al., Progression to AIDS, a clinical AIDS condition, and mortality: Psychosocial and physiological predictors. Psychol. Med. 2002; 32: 1059-1073.

Lorenza C, Cibele C, Mark G. Quality of life among HIV-infected patients in brazil after initiation of treatment. Clinics. 2009; 64(9): 867-75.

Louwagie GM, Bachmann MO, Meyer K, Booysen Fle R, Fairal LR, Heunis C. Highly active antiretroviral treatment and health related quality of life in South African adults with human immunodeficiency virus infection: A cross-sectional analytical study. BMC Public Health. 2007; 7: 244.

Mónica B, Liliane L, Irismar O, et al., Quality of life, anxiety and depression in patients with HIV/AIDS who present poor adherence to antiretroviral therapy: across-sectional study in Salvador, Braz J Infectdis. 2017; 21(5): 507-514.

Moore RD, Chaisson RE. Natural history of HIV infection in the era of combination ant ire tro viral therapy. AIDS. 1999 Oct 1; 13(14): 1933-42.

Moore RD, Chaisson RE. Natural history of HIV infection in the era of combination anti retro viral therapy. AIDS. 1999 Oct1; 13(14): 1933-42. 
Mukesh S, Monika A, Jai S, et al., Anxiety among people living with HIV/AIDS on antiretro viral treatment at ending tertiary care hospitals in Lucknow, Utar Pradesh, India. Int J Res Med Sci. 2016; 4(7): 2897-2901.

Murri R, Fantoni M, Del Borgoc, et al., Determinants of Health-related quality of life in HIV -infected patients. AIDS Care. 2003 Aug; 15(4): 581-90.

NACO, Ministry of Health and Family Welfare, Government of India. India HIV Estimations. 2015. Technical Report. New Delhi. 2016. p. 3.

National Agency for Control of AIDS. Women, Girls and HIV in Nigeria Fact Sheet 2011; NACA 2012; Abuja.

National AIDS Control Organisation, Department of AIDS Control. Annual Report 2013-2014. New Delhi: Ministry of Health and Family Welfare, Government of India; 2014. p. 9-1 2.

Nicholas PK, Kirksey KM, Corless IB, Kemppainen J. Lipodystrophy and quality of life in HIV: symptom management issues. Applied nursing research: ANR. 2005; 18(1): 55 \pm 8 .

NIMH. HIV/AIDS and mental health. Maryland: National Institute of Mental Health (NIM H), 2016.

Nyongesa MK, Mwangala PN, Mwangi P, et al., Neurocognitive and mental health outcomes and association with quality of life among adults living with HIV: a cross-sectional focus on a low-literacy population from coastal Kenya. BMJ Open 2018; 8: e023914.

Ranjeeta T, Ronele B, Abigail H, et al., On behalf of the HPTN071 (PopART) Study Team* Differences in healthrelated quality of life between HIVpositive and HIV-negative people in Zambia and South Africa: a crosssectional baseline survey of the HPTN071 (PopA RT) trial. Lancet Glob Health. 2017; 5: e1133-41.
Rena M, Zhang Y, Pan K, et al., Assessment of Health-Related Quality of Life among People Living with HIV in Xinjiang, West China. Journal of the International Association of Providers of AIDS Care. 2017; 16(6): 588-594.

Rodkjaer L, Laursen T, Bale N, Sodeman M. Depression in patients with HIV is under-diagnosed: a cross-sectional study in Denmark. British HIV Assoc HIV Med. 2010; 11: 46-53.

Ruggles KV, Fang Y, Tate J, et al., What are the Paterns between depression, smoking, unhealthy alcohol use, and other substanceuse among individuals receiving medical care? A longitudinal study of 5479 Participants. AIDS Behav. 2017; 21(7): 2014-22.

Sadock BJ, Sadock VA. Kaplan and Sadock's Pocket Handbook of Clinical Psychiatry. Wiliams \& Wilkins, Philadelphia, PA, 2005; 193-227.

Saylor D, Dickens AM, Sacktor N, et al., HIV-associated neurocognitive disorder-pathogenesis and prospects for treatment. Nat Rev Neurol 2016; 12: 234-48.

Schroecksnadel K, Sarcleti M, Winkler C et al., Quality of life and immune activation in patients with HIV infection. Brain Behav. Immun. 2008; 22: 881-889.

Sheline YI. 3DMRI studies of neuroanatomic changes in unipolar major depression: The role of stress and medical comorbidity. Biol. Psychiatry 2000; 48: 791-800.

Signh D. What is in name? AIDS dementia complex, AIDS dementia complex, HIV associated dementia, HIV associated neurocognitive disorder or HIV encephalopathy. Afr J Psychiatry. 2012; 15(3): 172-175.

Sow PS, Otieno LF, Bissagnene E, et al., Implementation of an antiretroviral access program for HIV-1-infected 
individuals in resource-limited setings: Clinical results from 4 African countries. J Acquir Immune Defic Syndr. 2007; 44(3): 262-267.

Tozzi V, Balestra P, Murri R, et al., Neurocognitive impairment influences quality of life in HIV-infected patients receiving HAART. IntJSTDAIDS 2004; 15: 254-9.

Vivithanaporn P, Heo G, Gamble J, et al., Neurologic disease burden in treated HIV/AIDS predicts survival: a population-based study. Neurology 2010; 75: 1150-8.

Watkins CC, Treisman GJ. Cognitive impairment in patients with AIDSprevalence and severity. HIV AIDS, 2015; 7: 35-47.

WHO. Global summary of the AIDS epidemic, 2013. 2014. Retrieved June 24, 2015, 2014, from htp://www.who.int/hiv/data/epicore_dec 2014.png?ua=1 (2017) Global AIDS Update 2017. AIDS info website.

Wig N, Lekshmi R, Pal H, Ahuja V Mital CM, Agarwal SK. The impact of HIV/AIDS on the quality of life: A cross-sectional study in north India. Indian J Med Sci. 2006 Jan; 60(1): 3-12.

Zhu T, Muthui D, Holte S, et al.: Evidence for human immunodeficiency virus type 1 replication invivo in CD14+ monocytes and its potential role as a source of virus in patients on highly active antiretroviral therapy. J Virol. 2002; 76(2): 707-716.

\section{How to cite this article:}

Vinay Malik, A.P. Garg, Tung Virsingh Arya and Maya Datt Joshi. 2019. Quality of Life with Mental Health HIV-Infected Patients in India before and After Antiretroviral Therapy (ART). Int.J.Curr.Microbiol.App.Sci. 8(06): 3321-3337. doi: https://doi.org/10.20546/ijcmas.2019.806.396 